\title{
Comparative Study of Fourteen Alkaloids from Uncaria rhynchophylla Hooks and Leaves Using HPLC-Diode Array Detection-Atmospheric Pressure Chemical Ionization/MS Method
}

\author{
Jialin Qu, ${ }^{a}$ Tianxing Gong, ${ }^{b}$ Bin Ma, ${ }^{a}$ Lin Zhang, ${ }^{a}$ Yoshihiro Kano, ${ }^{a}$ and Dan Yuan ${ }^{*}, a$ \\ ${ }^{a}$ School of Traditional Chinese Medicine, Shenyang Pharmaceutical University; 103 Wenhua Rd., Shenyang 110016, \\ China: and ${ }^{b}$ University of British Columbia; 2329 West Mall, Vancouver, B.C. V6T 1Z1, Canada. \\ Received June 20, 2011; accepted October 4, 2011; published online November 4, 2011
}

\begin{abstract}
The purpose of the study is to compare alkaloid profile of Uncaria rhynchophylla hooks and leaves. Ten oxindole alkaloids and four glycosidic indole alkaloids were identified using HPLC-diode array detection (DAD) or LC-atmospheric pressure chemical ionization (APCI)-MS method, and a HPLC-UV method for simultaneous quantification of major alkaloids was validated. The hooks are characterized by high levels of four oxindole alkaloids rhynchophylline (R), isorhynchophylline (IR), corynoxeine (C) and isocorynoxeine (IC), while the leaves contained high level of two glycosidic indole alkaloids vincoside lactam (VL) and strictosidine (S). The presented methods have proven its usefulness in chemical characterization of $U$. rhynchophylla hooks and leaves.
\end{abstract}

Key words oxindole alkaloid; glycosidic alkaloid; Uncaria rhynchophylla; alkaloid profile; HPLC; LCatmospheric pressure chemical ionization-MS

China has an abundant resource of Uncaria rhynchophylla (MiQ.) JACKS. The hooks on this plant are used in the treatment of infantile convulsion, headache, dizziness, hypertension and stroke in Traditional Chinese Medicine and Japanese Kampo Medicine. ${ }^{1,2)}$ In addition, the leaves, as a candidate part of the hooks, ${ }^{3)}$ are used in the manufacture of an antihypertensive remedy in China. ${ }^{4)}$ Oxindole alkaloids such as rhynchophylline (R) and isorhynchophylline (IR) are major components of $U$. rhynchophylla, ${ }^{5)}$ responsible for the cardiocerebral vascular effects, including hypotension, ${ }^{6)}$ vasodilation, ${ }^{7)}$ anti-platelet aggregation ${ }^{8)}$ and protective effect against neuronal damage. ${ }^{9)}$

Concerning quality assessment of $U$. rhynchophylla hooks, HPLC-UV method may be practical for determination of oxindole alkaloids due to the characteristic absorption of indole ring at $245 \mathrm{~nm}$ and $290 \mathrm{~nm} .{ }^{10)}$ The alkaloid profile of the hooks was studied using HPLC-UV technique in the previous literatures. ${ }^{11)}$ LC-MS technique is able to provide both analytical separation and structural determination of unknown bioactive compounds, which was applied to chemically characterize the oxindole alkaloids from Uncaria tormentosa ${ }^{12)}$ and the metabolites of $\mathrm{R}$ and IR. ${ }^{13,14)}$ Yamanaka et al. determined ten tertiary alkaloids in various parts of U. rhynchophylla, indicating that the alkaloid profile of the leaves is similar to that of the hooks. ${ }^{15)}$ Recently, we reported the isolation of several oxindole alkaloids and glycosidic indole alkaloids from $U$. rhynchophylla leaves, together with their inhibitory effects on microglial activation. R, IR, corynoxeine (C), isocorynoxeine (IC), strictosidine (S) and vincoside lactam (VL) are found to be potently active. ${ }^{16-18)}$ However, the chemical analysis of $\mathrm{S}$ and VL of $U$. rhynchophylla has not been reported yet.

In the present study, ten oxindole alkaloids and four glycosidic alkaloids from $U$. rhynchophylla hooks and leaves were identified by HPLC-diode array detection (DAD) and LC-MS technologies. Moreover, an accurate and sufficiently sensitive HPLC-UV method was validated to simultaneously determine these alkaloids. Utilizing optimized methods, the comprehensive alkaloid profiles of the hooks and leaves of $U$. rhyncho- phylla have been clarified.

\section{Experimental}

Chemicals and Reagents The standard compounds of fourteen alkaloids used in the qualitative and quantitative determination were isolated from $U$. rhynchophylla leaves in our previous studies. ${ }^{16,17)}$ They included ten oxindole alkaloids, 18,19-dehydrocorynoxinic acid (DCA), 18,19-dehydrocorynoxinic acid B (DCAB), isocorynoxeine $N$-oxide (IC-NO), corynoxeine $\mathrm{N}$-oxide (C-NO), isorhynchophylline $N$-oxide (IR$\mathrm{NO}$ ), rhynchophylline $N$-oxide (R-NO), IC, IR, C and R, and four glycosidic alkaloids, 11-hydroxy-2'-O-D-glucopyranosyl vincoside lactam (HGVL), 22- $O-\beta$-D-glucopyranosyl isocorynoxeinic acid (GICeA), S and VL (see Fig. 1). The identity of these compounds was confirmed by melting point, UV, IR, ${ }^{1} \mathrm{H}$ - and ${ }^{13} \mathrm{C}-\mathrm{NMR}$ and MS. ${ }^{16,17)}$ Their purities that were evaluated with HPLC-UV were more than 95\%. Other chemical reagents were of analysis or HPLC grades. MQ-water daily prepared with a Milli-Q water purification system (Millipore, Molsheim, France) was used in the mobile phase.

Plant Materials Eight aerial samples were directly collected from some growing regions in China (see Table 6 for details), all of which were authenticated as Uncaria rhynchophylla (MiQ.) JACKS. by Professor Zerong Jiang, Shenyang Pharmaceutical University, China. Voucher specimens were kept in the reference library with the medicinal herbs at Shenyang Pharmaceutical University. The hook and leaf samples were separated from the aerial parts in our laboratory, respectively.

Instrumentation and Chromatographic Conditions Qualitative and quantitative analyses were performed on a Kromasil $\mathrm{C}_{18}$ column $(4.6 \times 200 \mathrm{~mm}, 5 \mu \mathrm{m}$, Tianjin Scientific Instruments Co., Ltd., China) at $45^{\circ} \mathrm{C}$. The mobile phase consisted of a gradient system of solution A, methanol containing $0.03 \%$ diethylamine, and solution $\mathrm{B}$, water containing $0.03 \%$ diethylamine, at the flow rate of $1.0 \mathrm{~mL} / \mathrm{min}$. The gradient program was as follows: solution $\mathrm{A}-\mathrm{B}$ by linear gradient from $30: 70(\mathrm{v} / \mathrm{v})$ to $36: 64(\mathrm{v} / \mathrm{v})$ in $6 \mathrm{~min}$, held at $36: 64(\mathrm{v} / \mathrm{v})$ 


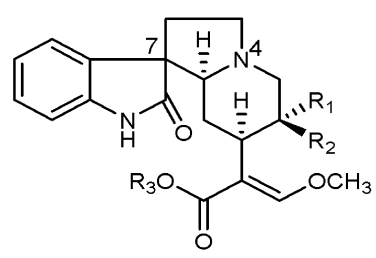

\begin{tabular}{lccccc} 
Comp. & $\mathrm{C}-7$ & $\mathrm{~N}-4$ & $\mathrm{R}_{1}$ & $\mathrm{R}_{2}$ & $\mathrm{R}_{3}$ \\
\hline DCAB & $R$ & tertiary & $-\mathrm{H}$ & $-\mathrm{CH}=\mathrm{CH}_{2}$ & $-\mathrm{H}$ \\
DCA & $\mathrm{S}$ & tertiary & $-\mathrm{H}$ & $-\mathrm{CH}=\mathrm{CH}_{2}$ & $-\mathrm{H}$ \\
$\mathrm{C}$ & $R$ & tertiary & $-\mathrm{CH}=\mathrm{CH}_{2}$ & $-\mathrm{H}$ & $-\mathrm{CH}_{3}$ \\
$\mathrm{IC}$ & $\mathrm{S}$ & tertiary & $-\mathrm{CH}=\mathrm{CH}_{2}$ & $-\mathrm{H}$ & $-\mathrm{CH}_{3}$ \\
R & $R$ & tertiary & $-\mathrm{CH}_{2} \mathrm{CH}_{3}$ & $-\mathrm{H}$ & $-\mathrm{CH}_{3}$ \\
IR & $S$ & tertiary & $-\mathrm{CH}_{2} \mathrm{CH}_{3}$ & $-\mathrm{H}$ & $-\mathrm{CH}_{3}$ \\
GICeA & $\mathrm{S}$ & tertiary & $-\mathrm{CH}=\mathrm{CH}_{2}$ & $-\mathrm{H}$ & $-\mathrm{GlC}$ \\
C-NO & $R$ & $N$-oxide & $-\mathrm{CH}=\mathrm{CH}_{2}$ & $-\mathrm{H}$ & $-\mathrm{CH}_{3}$ \\
IC-NO & $\mathrm{S}$ & N-oxide & $-\mathrm{CH}=\mathrm{CH}_{2}$ & $-\mathrm{H}$ & $-\mathrm{CH}_{3}$ \\
R-NO & $R$ & $N$-oxide & $-\mathrm{CH}_{2} \mathrm{CH}_{3}$ & $-\mathrm{H}$ & $-\mathrm{CH}_{3}$ \\
IR-NO & $S$ & $N$-oxide & $-\mathrm{CH}_{2} \mathrm{CH}_{3}$ & $-\mathrm{H}$ & $-\mathrm{CH}_{3}$ \\
\hline
\end{tabular}

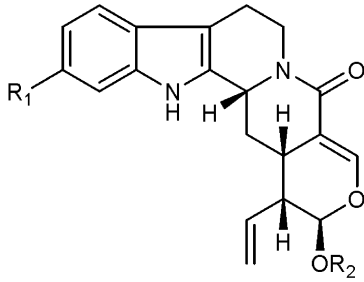

\begin{tabular}{lcc} 
Comp. & $\mathrm{R}_{1}$ & $\mathrm{R}_{2}$ \\
\hline $\mathrm{HGVL}$ & $-\mathrm{OH}$ & $-\mathrm{Glc}-\mathrm{Glc}$ \\
VL & $-\mathrm{H}$ & $-\mathrm{Glc}$ \\
\hline
\end{tabular}

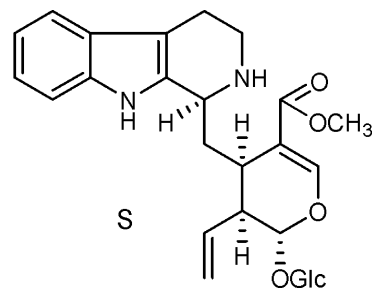

Fig. 1. Alkaloid Compounds Identified in U. rhynchophylla Hooks and Leaves

in $8 \mathrm{~min}$, by linear gradient to $45: 55(\mathrm{v} / \mathrm{v})$ in $11 \mathrm{~min}$, to $50: 50$ $(\mathrm{v} / \mathrm{v})$ in $25 \mathrm{~min}$, and finally to $65: 35(\mathrm{v} / \mathrm{v})$ in $35 \mathrm{~min}$. The injection volume was $10 \mu \mathrm{L}$.

Alkaloid identification by LC-MS was preformed on Shimadzu QP8000 $\alpha$ LC-MS spectrometer equipped with atmospheric pressure chemical ionization (APCI) interface (Shimadzu, Japan). APCI-MS was performed in the positive or negative modes under the following operating parameters: scanning mode: total ion current (TIC)/selective ion monitoring (SIM), scanning range $(\mathrm{m} / \mathrm{z}): 100-1000$, capillary voltage: $1.8 \mathrm{kV}$, CDL temperature: $250^{\circ} \mathrm{C}$, APCI temperature: $400^{\circ} \mathrm{C}$, flow rate of dry nitrogen: $2.5 \mathrm{~L} / \mathrm{min}$. The online UV spectra were obtained with a Waters HPLC system (Waters, U.S.A.) consisting of model 510 pump, automated gradient controller, model 2996 photodiode array detector, and Millennium32 software. They were recorded in the range of $200-400 \mathrm{~nm}$.

Shimadzu LC-2010AHT HPLC system equipped with CLASS-VP workstation (Shimadzu, Japan) was used for quantitative determinations, and the detective wavelength was set at $245 \mathrm{~nm}$.

Preparation of Standard Solutions Primary stock solutions of fourteen alkaloid references at a final concentration of $0.2 \mathrm{mg} / \mathrm{mL}$ were separately prepared by dissolving the accurately weighed reference compounds in $\mathrm{MeOH}-\mathrm{CHCl}_{3}$ $(70: 30)$. The stock solutions were then serially diluted until desired concentrations to obtain calibration curves. All of the solutions were stored at $4^{\circ} \mathrm{C}$ prior to use.

Sample Preparation The ground plant materials $(0.3 \mathrm{~g}$, passing through a $500 \mu \mathrm{m}$ mesh sieve) were double-extracted in $\mathrm{MeOH}-\mathrm{H}_{2} \mathrm{O}(70: 30,30 \mathrm{~mL}$ each) by sonication at room temperature for $20 \mathrm{~min}$. The mixtures were centrifuged at $3000 \mathrm{rpm}$ for $10 \mathrm{~min}$. The combined supernatant and washing liquid were evaporated to dryness in vacuo. The residues were reconstituted in $10 \mathrm{~mL}$ of $\mathrm{MeOH}-\mathrm{CHCl}_{3}(70: 30)$, and then filtered through a $0.45 \mu \mathrm{m}$ membrane filter prior to use.

Stability Trial The reference standards VL, ICO, IR, CO, and $\mathrm{R}$ in proper amount were dissolved in $10 \mathrm{~mL}$ of $\mathrm{MeOH}$ or $\mathrm{MeOH}-\mathrm{CHCl}_{3}(70: 30)$ at the concentration of $0.1 \mathrm{mg} / \mathrm{mL}$, respectively. Their stability was determined during $30-\mathrm{d}$ storage at $4^{\circ} \mathrm{C}$. They were taken on day $8,16,24$ and 30 , and subsequently subjected to HPLC analysis. For the stability trial, the mobile phase consisting of the solutions A and B was run in isocratic mode in the ratio of 50:50 (v/v). Other HPLC conditions were the same as those described in the qualitative and quantitative determination.

The concentrations of five standard solutions were calculated using the following calibrations, R: $y=1.790 \times 10^{7} x+3.509 \times 10^{5}$ $(r=0.9993)$, IR: $y=1.922 \times 10^{7} x-9.464 \times 10^{4} \quad(r=1.000), \mathrm{C}: y=$ $2.192 \times 10^{7} x+2.399 \times 10^{4}(r=0.9993)$, IC: $y=1.435 \times 10^{7} x+8.471 \times$ $10^{5}(r=1.000)$, VL: $y=1.457 \times 10^{7} x-1.885 \times 10^{5}(r=0.9994)$. The linear range for these calibrations was from $0.04 \mathrm{mg} / \mathrm{mL}$ to $0.16 \mathrm{mg} / \mathrm{mL}$. The control data described as day 0 was obtained from the samples just before the stability trial.

Data Analysis Each sample solution was injected in triplicate, and the content of the analytes was calculated according to the corresponding calibration curves. The full identification of all marker compounds was performed by comparing the retention time, MS fragments, and UV spectrum of their peaks with corresponding standards.

\section{Results and Discussion}

Identification of Fourteen Alkaloids by HPLC-DAD and LC-APCI-MS Methods In order to characterize the major alkaloids from $U$. rhynchophylla, a hook samples (Sample \#1) were subjected to HPLC-UV and LC-MS analyses (see Fig. 2). Fourteen specific peaks (labeled Peaks 1-14, Fig. 2) were characterized by typical UV absorptions of oxindole ring at $242-249 \mathrm{~nm}$ or indole ring at $281-296 \mathrm{~nm}$. By direct comparison of the characteristic molecular ions, UV spectra as well as retention time with those of authentic standards, the alkaloids corresponding to Peaks $1-14$ were identified as DCA, DCAB, HGVL, IC-NO, C-NO, IR-NO, R-NO, GICeA, $\mathrm{S}, \mathrm{VL}, \mathrm{IC}, \mathrm{IR}, \mathrm{C}$ and R, respectively (see Table 1). They were satisfactorily separated under the HPLC-UV condition. The detective wavelength for quantification of these alkaloids was chosen at $245 \mathrm{~nm}$ by comparison of the chromatograms of sample solutions at different wavelengths.

The APCI-MS analysis was operated in TIC and SIM scan 
modes detecting positive or negative ions. As shown in Fig. 2, for the oxindole alkaloids with one basic nitrogen atom, the positive-ion mode produced a stronger ion current. Glycosidic alkaloids with acidic hydroxyl groups gave stronger negative ions. Overall, the signal intensity of all the alkaloids in LCAPCI-MS chromatograms was stronger than that achievable from UV detector. Since background level running in APCISIM mode is far less, SIM analysis allowed for minor alkaloids such as DCA, DCAB, IC-NO, C-NO, IR-NO, R-NO and

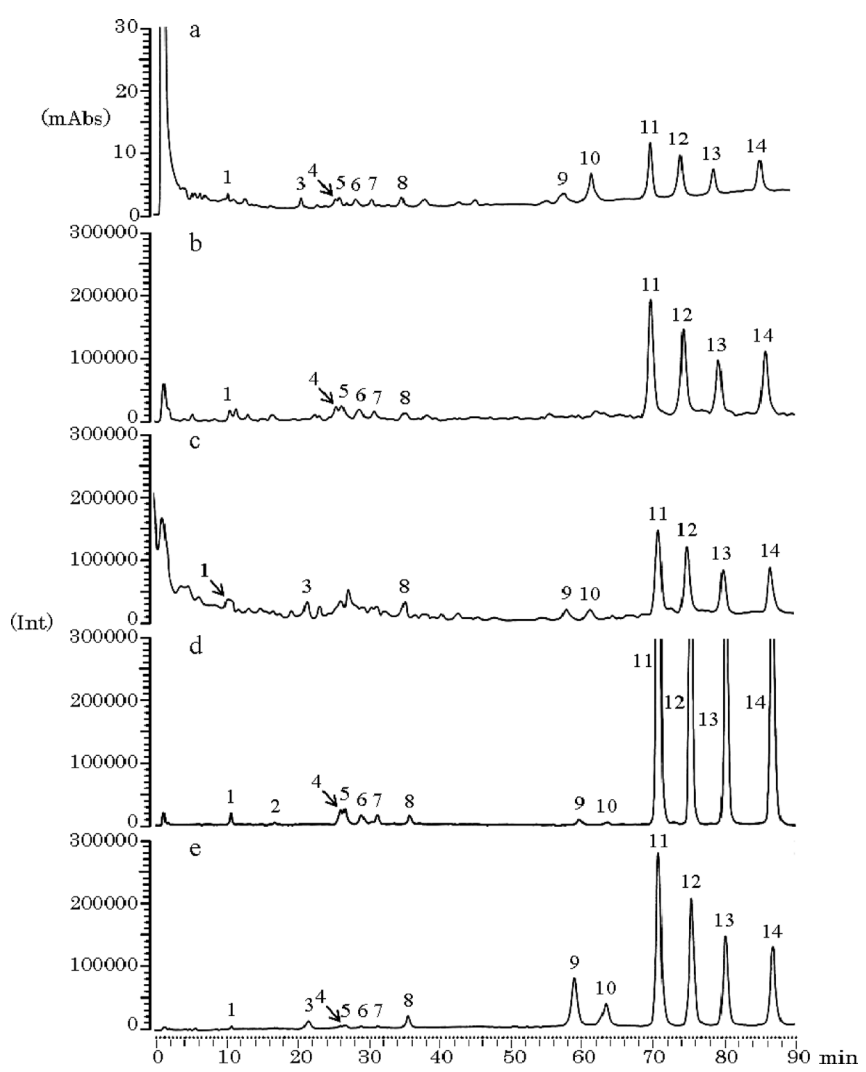

Fig. 2. Chromatograms of Alkaloids Occurring in U. rhynchophylla Hooks (Sample \#1)

(a) HPLC chromatogram detected at $245 \mathrm{~nm}$. (b) and (c) TIC chromatograms in positive and negative ion modes. (d) and (e) SIM chromatograms in positive and negative ion modes. See Table 1 for the peak numbers, and see Experimental section for HPLC-MS conditions.
GICeA to be efficiently detected and measured.

Extraction of Marker Alkaloids In order to optimize the extraction of various alkaloids from the plant materials, we compared several extractive procedures. As shown in Table 2, a direct ultrasonic extraction for 20 min with $\mathrm{MeOH}-\mathrm{H}_{2} \mathrm{O}$ (70:30) resulted in a more effective extraction of IC and $\mathrm{C}$ and a similar extraction of VL, IR and $\mathrm{R}$ from U. rhynchophylla leaf (Sample \#12), compare with the same extractive manner but with $\mathrm{MeOH}$ or $\mathrm{MeOH}-10 \% \operatorname{HOAc}(70: 30) .{ }^{19)}$ Also a reflux extraction with $\mathrm{MeOH}-\mathrm{H}_{2} \mathrm{O}(70: 30)$ resulted in similar extraction of major alkaloids to an ultrasonic extraction plus a successive reflux extraction with the same solvent. This result indicates that a great increase in the amount of VL, C and $\mathrm{R}$ resulted from the reflux extraction should be ascribed to the transformation or isomerization of the alkaloid compounds during the heat process. $^{20)}$ A single ultrasonic extraction with $\mathrm{MeOH}-\mathrm{H}_{2} \mathrm{O}(70: 30)$ is, therefore, preferred to oxindole and glycosidic indole alkaloids.

The isomerization of rhynchophylline-type alkaloids occurs at spiro center C-7 during the heat process, or in alkalinic or polar solvents. ${ }^{21)}$ In order to understand the stability of these alkaloid compounds and to select a proper solvent for inhibition of their isomerization, we undertook a stability trial for five reference standards VL, IC, IR, C and R. They were dissolved in $\mathrm{MeOH}$ or $\mathrm{MeOH}-\mathrm{CHCl}_{3}(70: 30)$, respectively, and subsequently analyzed during $30 \mathrm{~d}$ of storage at $4^{\circ} \mathrm{C}$. The representative HPLC chromatograms of the stability trial are shown in Fig.3, and the course of the isomerization is depicted in Fig. 4. The content levels of R, IR, C and IC decreased $17.6 \%, 8.1 \%, 7.1 \%, 9.2 \%$ in $\mathrm{MeOH}$, respectively, on day 30 after storage at $4^{\circ} \mathrm{C}$, while they were rather stable in $\mathrm{MeOH}-$ $\mathrm{CHCl}_{3}$ (70:30) under the same storage condition. The decomposition products of the four alkaloids, which were identified by their UV spectra as well as co-chromatographic procedures with the reference standards, were their respective 7-configurational isomers. The $7 S$ isomers seem to be more stable than the $7 R$ isomers. The result of stability trial is consistent with Laus's study. ${ }^{21)}$ In addition, VL retained stable content level in both $\mathrm{MeOH}$ and $\mathrm{MeOH}-\mathrm{CHCl}_{3}$ (70:30). $\mathrm{MeOH}-\mathrm{CHCl}_{3}$ $(70: 30)$ is, therefore, a preferable solvent for these oxindole alkaloids.

Validation of HPLC Method Good linear calibration

Table 1. The Alkaloids Identified from U. rhynchophylla Hooks and Leaves by HPLC-DAD and LC-APCI-MS Methods

\begin{tabular}{|c|c|c|c|c|}
\hline Compounds (Peak No.) & $t_{\mathrm{R}}(\min )$ & $\mathrm{UV}\left(\lambda_{\max }, \mathrm{nm}\right)$ & Molecular ion $(\mathrm{m} / \mathrm{z})$ & MS approach \\
\hline DCA (1) & 10.6 & 210,242 & $369.1[\mathrm{M}+\mathrm{H}]^{+}$ & TICp, SIM \\
\hline DCAB (2) & 15.5 & 212,242 & $369.1[\mathrm{M}+\mathrm{H}]^{+}$ & SIMp \\
\hline HGVL (3) & 21.0 & 227,296 & $675.0[\mathrm{M}-\mathrm{H}]^{-}$ & TICn, SIMn \\
\hline IC-NO (4) & 25.3 & 210,247 & $401.1[\mathrm{M}+\mathrm{H}]^{+}$ & TICp, SIM \\
\hline $\mathrm{C}-\mathrm{NO}(\mathbf{5})$ & 26.0 & 210,247 & $401.1[\mathrm{M}+\mathrm{H}]^{+}$ & TICp, SIM \\
\hline IR-NO (6) & 28.1 & 210,248 & $398.9[\mathrm{M}+\mathrm{H}]^{+}$ & TICp, SIM \\
\hline R-NO (7) & 30.2 & 208,243 & $399.1[\mathrm{M}+\mathrm{H}]^{+}$ & TICp, SIM \\
\hline $\mathrm{GICeA}(\mathbf{8})$ & 34.7 & 210,249 & $529.1[\mathrm{M}-\mathrm{H}]^{-}$ & TIC, SIM \\
\hline $\mathrm{S}(\mathbf{9})$ & 57.9 & 228,281 & $528.8[\mathrm{M}-\mathrm{H}]^{-}$ & TICn, SIM \\
\hline VL (10) & 61.8 & 228,282 & $497.0[\mathrm{M}-\mathrm{H}]^{-}$ & TICn, SIM \\
\hline IC (11) & 69.0 & 208,245 & $383.1[\mathrm{M}+\mathrm{H}]^{+}$ & TIC, SIM \\
\hline IR (12) & 73.1 & 209,245 & $385.1[\mathrm{M}+\mathrm{H}]^{+}$ & TIC, SIM \\
\hline $\mathrm{C}(\mathbf{1 3})$ & 77.9 & 209,245 & $382.9[\mathrm{M}+\mathrm{H}]^{+}$ & TIC, SIM \\
\hline R (14) & 84.1 & 210,247 & $385.1[\mathrm{M}+\mathrm{H}]^{+}$ & TIC, SIM \\
\hline
\end{tabular}


Table 2. Optimization of Extraction of Major Alkaloids

\begin{tabular}{|c|c|c|c|c|c|}
\hline \multirow{2}{*}{$\begin{array}{l}\text { Extractive procedures, solvents, time and } \\
\text { temperature }\end{array}$} & \multicolumn{5}{|c|}{ Content (mg/g) } \\
\hline & VL & IC & IR & $\mathrm{C}$ & $\mathrm{R}$ \\
\hline \multicolumn{6}{|l|}{ Ultrasonic $/ 20^{\circ} \mathrm{C} / 20 \mathrm{~min}$} \\
\hline $\mathrm{MeOH}$ & 2.62 & 1.07 & 1.02 & 0.58 & 0.56 \\
\hline $\mathrm{MeOH}-\mathrm{H}_{2} \mathrm{O}(70: 30)$ & 2.62 & 1.26 & 1.24 & 0.66 & 0.59 \\
\hline $\mathrm{MeOH}-10 \%$ HAC $(70: 30)$ & 2.61 & 1.22 & 1.26 & 0.65 & 0.59 \\
\hline \multicolumn{6}{|l|}{$\mathrm{MeOH}-\mathrm{H}_{2} \mathrm{O}(70: 30)$} \\
\hline Reflux $/ 90^{\circ} \mathrm{C} / 20 \mathrm{~min}$ & 4.39 & 0.98 & 1.05 & 0.86 & 0.92 \\
\hline $\begin{array}{c}\text { Ultrasonic } / 20^{\circ} \mathrm{C} / 20 \mathrm{~min} \\
+\operatorname{reflux} / 90^{\circ} \mathrm{C} / 20 \mathrm{~min}\end{array}$ & 2.62 & 1.29 & 1.23 & 0.69 & 0.59 \\
\hline
\end{tabular}

Sample \#12 was used in the experiment.

curves and the linear range were obtained for all the alkaloid standards except for DCAB and C-NO (see Table 3). The $r$ values were more than $0.999-1.000$. The calibrations for

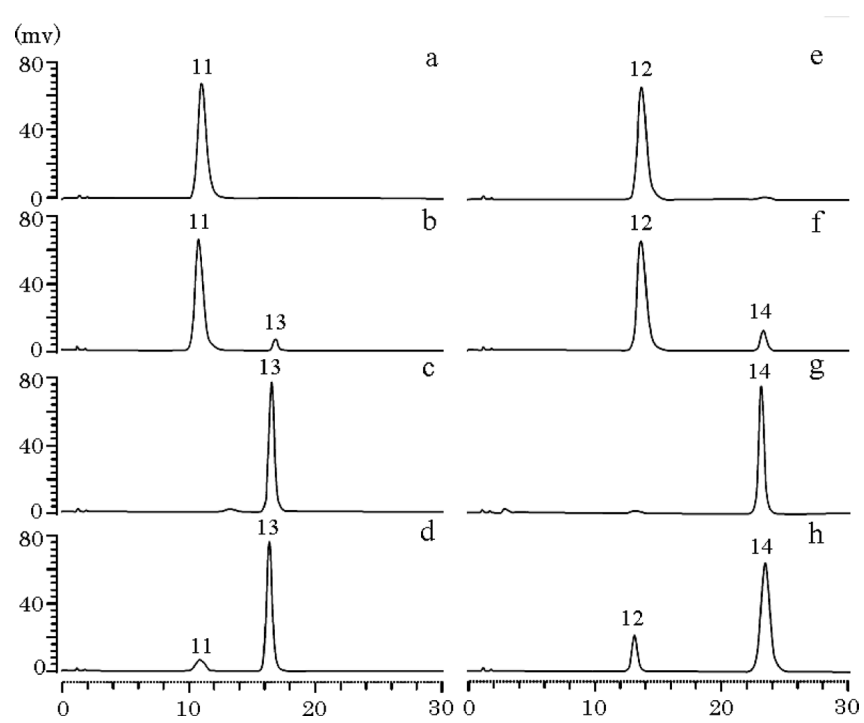

Fig. 3. HPLC Chromatograms of Freshly Prepared $\mathrm{MeOH}-\mathrm{CHCl}_{3}$ (70:30) Solution of IC (a), C (c), IR (e) and R (g), and the Solutions on Day 30 of Storage at $4^{\circ} \mathrm{C}(\mathrm{b}, \mathrm{d}, \mathrm{f}, \mathrm{h})$

The four tested solution are at the concentration of $0.1 \mathrm{mg} / \mathrm{mL}$. See Table 1 for the peak numbers, and see stability trial of Experimental for HPLC condition.
DCAB was not established because it was either absent or present in trace amounts in all the samples. Because of a great difference in the contents of S and VL between the hooks and leaves, two calibrations were established to serve for the low and high content levels.

The limit of detection $(S / N=3)$ of these alkaloid compounds were $c a$. $0.09-0.28 \mu \mathrm{g} / \mathrm{mL}$. The limit of quantitation $(S / N=$ 10) of these alkaloid compounds were $c a .0 .31-0.74 \mu \mathrm{g} / \mathrm{mL}$. The precision and recovery tests were done only for major alkaloid compounds HGVL, S, VL, IC, IR, C and R. This method had good reproducibility with intra-day variations (RSD) of less than $1.3 \%$ and inter-day variations (RSD) of less than $2.1 \%$ (see Table 4), respectively. Recovery data was obtained from the comparison of the responses between the test samples and the spiked samples. The recovery for the seven alkaloids ranged from 97.3 to $101.3 \%$, with RSD ranging from 0.9 to $2.9 \%$ (see Table 5). Thus, this analytical procedure is accurate and sufficiently sensitive for the quantitative determination of major alkaloids from U. rhynchophylla hooks and leaves.

Quantification of Thirteen Alkaloids of $U$. rhynchophylla Hooks and Leaves Chemical profiling and quantification of the thirteen alkaloids from $U$. rhynchophylla hooks and leaves using HPLC-UV method were carried out. Fig. 5 presents HPLC chromatograms of the methanolic extracts of a hook and a leaf as well as a mixture of fourteen alkaloid standards. Under the chromatographic condition, DCA (peak 1) may not

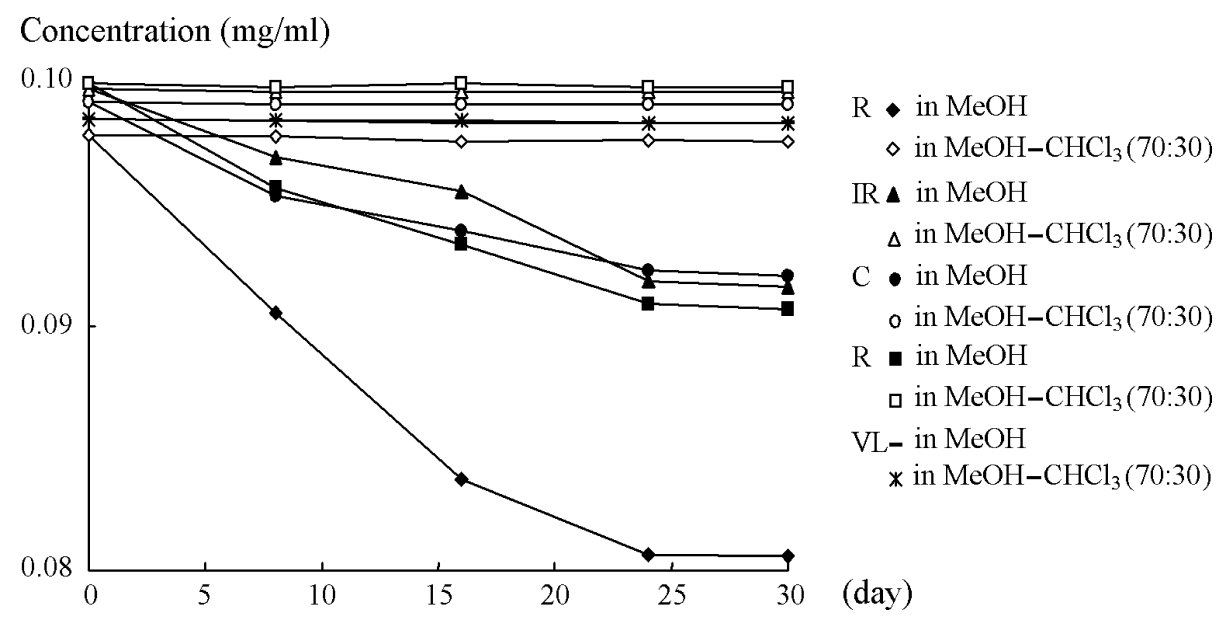

Fig. 4. Course of the Content Variations of VL, IC, IR, C and R in $\mathrm{MeOH}$ or $\mathrm{MeOH}-\mathrm{CHCl}_{3}(70: 30)$ during $30 \mathrm{~d}$ Storage at $4{ }^{\circ} \mathrm{C}$ For stability trials, see Experimental. 
Table 3. Calibration and Detection Limit for Twelve Alkaloids

\begin{tabular}{|c|c|c|c|c|c|}
\hline Compounds & Calibration curve & $r$ & Linear range $(\mathrm{mg} / \mathrm{mL})$ & $\operatorname{LOD}(\mu \mathrm{g} / \mathrm{mL})$ & LOQ $(\mu \mathrm{g} / \mathrm{mL})$ \\
\hline HGVL & $Y=1.073 \times 10^{7} x-7.682 \times 10^{4}$ & 0.999 & $4.30 \times 10^{-3}-1.70 \times 10^{-1}$ & 0.21 & 0.43 \\
\hline $\mathrm{IC}-\mathrm{NO}$ & $Y=1.996 \times 10^{7} x-5.788 \times 10^{3}$ & 0.999 & $4.25 \times 10^{-4}-8.50 \times 10^{-3}$ & 0.13 & 0.39 \\
\hline $\mathrm{C}-\mathrm{NO}$ & $Y=1.734 \times 10^{7} x-3.477 \times 10^{3}$ & 1.000 & $4.29 \times 10^{-4}-8.57 \times 10^{-3}$ & 0.12 & 0.37 \\
\hline IR-NO & $Y=1.963 \times 10^{7} x-5.364 \times 10^{3}$ & 1.000 & $3.80 \times 10^{-4}-7.60 \times 10^{-3}$ & 0.10 & 0.31 \\
\hline $\mathrm{R}-\mathrm{NO}$ & $Y=1.097 \times 10^{7} x-2.484 \times 10^{3}$ & 0.999 & $1.29 \times 10^{-3}-5.10 \times 10^{-3}$ & 0.09 & 0.37 \\
\hline GICeA & $Y=6.654 \times 10^{6} x-1.148 \times 10^{4}$ & 1.000 & $1.05 \times 10^{-3}-4.20 \times 10^{-3}$ & 0.14 & 0.33 \\
\hline \multirow[t]{2}{*}{$\mathrm{S}$} & $Y=3.127 \times 10^{6} x-2.790 \times 10^{4}$ & 0.999 & $8.10 \times 10^{-3}-1.61 \times 10^{-1}$ & 0.28 & 0.63 \\
\hline & $Y=4.526 \times 10^{6} x-3.417 \times 10^{5}$ & 0.999 & $8.07 \times 10^{-2}-1.61$ & & \\
\hline \multirow[t]{2}{*}{ VL } & $Y=1.657 \times 10^{7} x-1.376 \times 10^{5}$ & 0.999 & $8.80 \times 10^{-3}-1.75 \times 10^{-1}$ & 0.11 & 0.53 \\
\hline & $Y=1.372 \times 10^{7} x-9.850 \times 10^{5}$ & 0.999 & $8.75 \times 10^{-2}-1.75$ & & \\
\hline $\mathrm{IC}$ & $Y=2.190 \times 10^{7} x-2.923 \times 10^{4}$ & 0.999 & $3.50 \times 10^{-3}-1.40 \times 10^{-1}$ & 0.16 & 0.48 \\
\hline IR & $Y=2.001 \times 10^{7} x-1.061 \times 10^{4}$ & 1.000 & $2.50 \times 10^{-3}-9.92 \times 10^{-2}$ & 0.23 & 0.68 \\
\hline $\mathrm{C}$ & $Y=2.306 \times 10^{7} x-8.344 \times 10^{3}$ & 0.999 & $1.70 \times 10^{-3}-6.60 \times 10^{-2}$ & 0.18 & 0.55 \\
\hline $\mathrm{R}$ & $Y=1.780 \times 10^{7} x-1.378 \times 10^{3}$ & 0.999 & $3.27 \times 10^{-3}-6.54 \times 10^{-2}$ & 0.28 & 0.74 \\
\hline
\end{tabular}

LOD: limit of detection, LQD: limit of quantification.

Table 4. Intra- and Inter-Day Variations of the HPLC Method for Determination of Major Alkaloids

\begin{tabular}{cccc}
\hline \hline \multirow{2}{*}{ Compounds } & \multicolumn{2}{c}{ Intra-day precision } & Inter-day precision \\
\cline { 2 - 4 } & Content $(\mu \mathrm{g} / \mathrm{g})$ & RSD $(\%)$ & Content $(\mu \mathrm{g} / \mathrm{g})$ \\
\hline HGVL & $622 \pm 4$ & 0.57 & $620 \pm 12$ \\
S & $2305 \pm 30$ & 1.32 & $2291 \pm 43$ \\
VL & $3038 \pm 33$ & 1.10 & $3100 \pm 66$ \\
IC & $1933 \pm 5$ & 0.24 & $1923 \pm 30$ \\
IR & $1860 \pm 7$ & 0.37 & 1.88 \\
C & $655 \pm 4$ & 0.63 & 1.12 \\
R & $741 \pm 7$ & 1.00 & $659 \pm 13$ \\
\end{tabular}

Sample \#12 was used in the experiment.

be precisely quantified due to lack of the perfect detection. We found that the four oxinole alkaloids (IC, IR, C and R) and two glycosidic indole alkaloids (S and VL) are major components of hooks and leaves (see Fig. 5, Table 6). However, the content ratio of the oxindole alkaloids to the glycosidic alkaloids in both parts is quite different from each other. In the hooks, the total amount of four oxindole alkaloids (2.60$13.82 \mathrm{mg} / \mathrm{g})$ is much more than that of two glycosidic alkaloids $(0.15-1.50 \mathrm{mg} / \mathrm{g})$, while in the leaves, the total amount of the oxindole alkaloids $(2.18-7.11 \mathrm{mg} / \mathrm{g})$ is similar to that of the glycosidic alkaloids $(3.73-7.16 \mathrm{mg} / \mathrm{g})$. The data also demonstrated that two glycosidic indole alkaloids $\mathrm{S}$ and $\mathrm{VL}$ are in greater abundance in the leaves than in the hooks. Otherwise, DCAB, IC-NO, C-NO, IR-NO, R-NO and GICeA were not detected at all or detected in small or trace amounts in both the hooks and leaves. Several studies on HPLC analysis of oxindole alkaloids of Uncaria sp. are reported. ${ }^{22,23)}$ Most of them focused on RIN and IRN. Yamanaka et al. reported the HPLC analysis of four alkaloids (including IC, IR, C and R) and six indole alkaloids (including corynantheine, akuanmmigine, dihydrocorynoxeine, hirsuteine, hirsutine and geissoschizine methyl ether) in hooks and leaves of $U$. rhynchophylla, ${ }^{15)}$ indicating that the pattern of the ten alkaloids in hooks is similar to that in leaves. Additionally, HPLC-DAD or HPLC-ESI/MS determination of six penta-cyclic oxindole alkaloids, isopteropodin, pteropodine, isomitraphylline, uncarlne $\mathrm{F}$, milraphyllin, and speclophylline as well as IC, IR, $\mathrm{C}$ and $\mathrm{R}$ in U. tomentosa was reported. ${ }^{24)}$ In our present study, two glycosidic oxindole alkaloids, $\mathrm{S}$ and VL, were determined for the first time due to

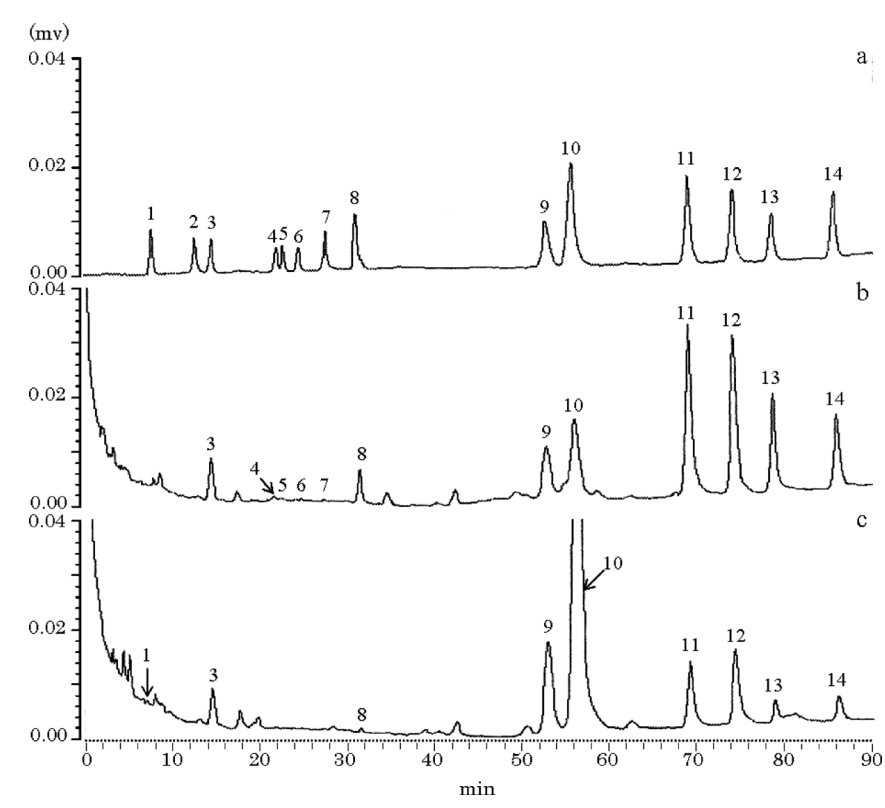

Fig. 5. HPLC Chromatograms of a Mixed Standard (a) and Typical Extracts of U. rhynchophylla Hooks (b, Sample \#1) and Leave (c, Sample \#2)

See Table 1 for the peak numbers, and see Experimental for quantification condition.

their existence in both hooks and leaves, together with four minor $\mathrm{N}$-oxide oxindole alkaloids.

In our previous study, IC, IR, C, R, S and VL demonstrated 
Table 5. Accuracy of the HPLC Method for Determination of Major Alkaloids

\begin{tabular}{|c|c|c|c|c|c|}
\hline Compounds & Added (mg) & Observed (mg) & Recovery (\%) & Mean (\%) & RSD (\%) \\
\hline \multirow[t]{6}{*}{ HGVL } & 0.1036 & 0.1018 & 98.3 & 99.5 & 1.6 \\
\hline & 0.1036 & 0.1028 & 99.2 & & \\
\hline & 0.1036 & 0.1060 & 102.3 & & \\
\hline & 0.1036 & 0.1041 & 100.5 & & \\
\hline & 0.1036 & 0.1020 & 98.5 & & \\
\hline & 0.1036 & 0.1018 & 98.3 & & \\
\hline \multirow[t]{6}{*}{ S } & 0.3820 & 0.3984 & 104.3 & 100.5 & 2.9 \\
\hline & 0.3820 & 0.3839 & 100.5 & & \\
\hline & 0.3820 & 0.3778 & 98.9 & & \\
\hline & 0.3820 & 0.3728 & 97.6 & & \\
\hline & 0.3820 & 0.3958 & 103.6 & & \\
\hline & 0.3820 & 0.3736 & 97.8 & & \\
\hline \multirow[t]{6}{*}{ VL } & 0.5080 & 0.4917 & 96.8 & 98.8 & 1.4 \\
\hline & 0.5080 & 0.5095 & 100.3 & & \\
\hline & 0.5080 & 0.4999 & 98.4 & & \\
\hline & 0.5080 & 0.5034 & 99.1 & & \\
\hline & 0.5080 & 0.5095 & 100.3 & & \\
\hline & 0.5080 & 0.4978 & 98.0 & & \\
\hline \multirow[t]{6}{*}{ IC } & 0.3195 & 0.3125 & 97.8 & 98.8 & 1.6 \\
\hline & 0.3195 & 0.3131 & 98.0 & & \\
\hline & 0.3195 & 0.3083 & 96.5 & & \\
\hline & 0.3195 & 0.3211 & 100.5 & & \\
\hline & 0.3195 & 0.3208 & 100.4 & & \\
\hline & 0.3195 & 0.3185 & 99.7 & & \\
\hline \multirow[t]{6}{*}{ IR } & 0.3333 & 0.3392 & 101.8 & 101.4 & 1.7 \\
\hline & 0.3333 & 0.3369 & 101.1 & & \\
\hline & 0.3333 & 0.3341 & 100.2 & & \\
\hline & 0.3333 & 0.3486 & 104.6 & & \\
\hline & 0.3333 & 0.3361 & 100.9 & & \\
\hline & 0.3333 & 0.3332 & 100.0 & & \\
\hline \multirow[t]{6}{*}{$\mathrm{C}$} & 0.1067 & 0.1085 & 101.7 & 101.3 & 0.9 \\
\hline & 0.1067 & 0.1093 & 102.4 & & \\
\hline & 0.1067 & 0.1082 & 101.4 & & \\
\hline & 0.1067 & 0.1068 & 100.1 & & \\
\hline & 0.1067 & 0.1085 & 101.7 & & \\
\hline & 0.1067 & 0.1069 & 100.2 & & \\
\hline \multirow[t]{6}{*}{$\mathrm{R}$} & 0.1217 & 0.1187 & 97.5 & 97.3 & 1.1 \\
\hline & 0.1217 & 0.1204 & 98.9 & & \\
\hline & 0.1217 & 0.1170 & 96.1 & & \\
\hline & 0.1217 & 0.1179 & 96.9 & & \\
\hline & 0.1217 & 0.1191 & 97.9 & & \\
\hline & 0.1217 & 0.1176 & 96.6 & & \\
\hline
\end{tabular}

Sample \#12 was used in the experiment.

the potent inhibitory activity against NO release from microglia, with the other minor alkaloids being of less or weak activity. ${ }^{16,17)}$ The comprehensive chromatographic profiles in this study demonstrate not only the similarity in the alkaloid composition but also the difference in quantities of the major components between the hooks and the leaves of $U$. rhynchophylla. Therefore, the leaves are a valuable candidate for the hooks due to the abundance in the active alkaloids as well as rich botanical nature.

\section{Conclusions}

The present study is the first report on HPLC-DAD and HPLC-APCI/MS methods for the chemical profiling of fourteen alkaloids from $U$. rhynchophylla hooks and leaves. Both $[\mathrm{M}+\mathrm{H}]^{+}$ions of oxindole alkaloids and $[\mathrm{M}-\mathrm{H}]^{-}$ions of glycosidic alkaloids, obtained by APCI-MS determination, showed higher signal intensity than their peaks achievable with HPLC-UV method, thus allowing a more efficient identification of these alkaloids from U. rhynchophylla. Moreover, a validated HPLC method was successfully applied for simultaneous determination of them with good accuracy and precision. The results demonstrate the similarity and differences in alkaloids composition between $U$. rhynchophylla hooks and leaves.

Acknowledgements This work was supported by the 2009 Scientific Technology Plan Project of Shenyang City Government (No. 1091134-9-00) and National Natural Sciences Foundation of China (No. 81173544). 


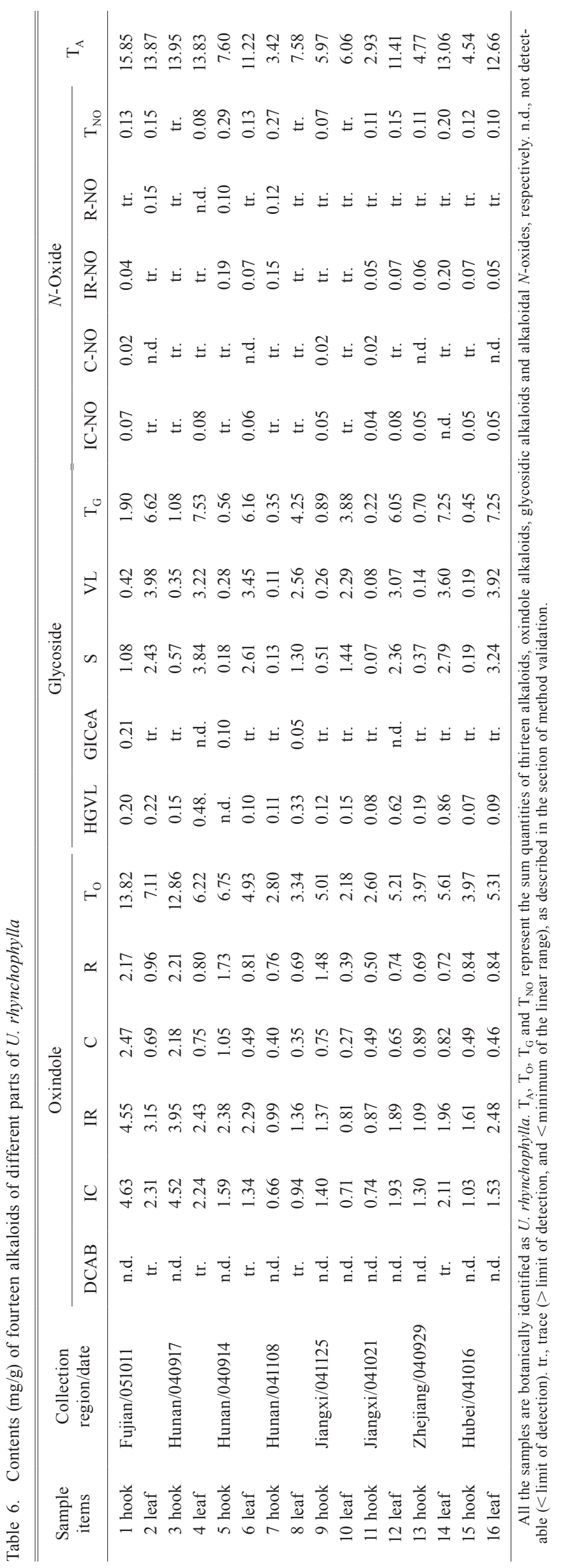




\section{References}

1) Bensky D., Gamble A., Kaptchuk T., "Chinese Herbal Medicine: Materia Medica," Revised ed., Eastland Press, Seattle, 1999, p. 423.

2) Shimada Y., Terasawa K., Yamamoto T., Maruyama I., Saitoh Y., Kanaki E., Takaori S., J. Tradit. Med., 11, 246-255 (1994).

3) The Health Bureau of Guangxi Autonomous Region Revolutionary Committee, "Guangxi Materia Medica Collection," Guangxi People's Press, China, 1974, p. 886.

4) State Food and Drug Adminstration of China, "Collection of Criterion for Traditional Chinese Medicine, Meridians," Limb and Brain System Vol., People's Medical Publishing House Press, China, 2002, p. 112.

5) Phillipson J. D., Hemingway S. R., Ridsdale C. E., Lloydia, 41, 503-570 (1978).

6) Shi J. S., Liu G. X., Wu Q., Huang Y. P., Zhang X. D., Zhongguo Yao Li Xue Bao, 13, 35-38 (1992).

7) Zhang W. B., Chen C. X., Sim S. M., Kwan C. Y., Naunyn Schmiedebergs Arch. Pharmacol., 369, 232-238 (2004).

8) Kang T. H., Murakami Y., Takayama H., Kitajima M., Aimi N., Watanabe H., Matsumoto K., Life Sci., 76, 331-343 (2004).

9) Shimada Y., Goto H., Itoh T., Sakakibara I., Kubo M., Sasaki H., Terasawa K., J. Pharm. Pharmacol., 51, 715-722 (1999).

10) Liu H. M., Feng X. Z., Phytochemistry, 33, 707-710 (1993).

11) Sakakibara I., Terabayashi S., Kubo M., Higuchi M., Sasaki H., Okada M., Nat. Med., 53, 308-312 (1999).
12) Lopez-Avila1V., Benedicto J., Robaugh D., J. High Resolut. Chromatogr., 20, 231-236 (1997).

13) Wang W., Ma C. M., Hattori M., Biol. Pharm. Bull., 33, 669-676 (2010).

14) Wang W., Ma C. M., Hattori M., J. Pharm. Pharm. Sci., 13, 27-37 (2010).

15) Yamanaka E., Kimizuka Y., Aimi N., Sakai S., Haginiwa J., Yakugaku Zasshi, 103, 1028-1033 (1983).

16) Yuan D., Ma B., Wu C. F., Yang J. Y., Zhang L. J., Liu S. K., Wu L. J., Kano Y., J. Nat. Prod., 71, 1271-1274 (2008).

17) Ma B., Wu C. F., Yang J. Y., Wang R., Kano Y., Yuan D., Helv. Chim. Acta, 92, 1575-1585 (2009).

18) Yuan D., Ma B., Yang J. Y., Xie Y. Y., Wang L., Zhang L. J., Kano Y., Wu C. F., Int. J. Immunopharmacol., 9, 1549-1554 (2009).

19) Society of Japanese Pharmacopoeia, "The Japanese Pharmacopoeia," 15th ed., Jiho, Tokyo, 2006, pp. 462-465.

20) Sakakibara I., Terabayashi S., Kubo M., Higuchi M., Komatsu Y., Okada M., Taki K., Kamei J., Phytomedicine, 6, 163-168 (1999).

21) Laus G., J. Chem. Soc., Perkin Trans. 2, 1998, 315-318 (1998).

22) Wang X. F., Wei L. X., Wang B. Z., Chin. J. Pharm. Anal., 19, 56-57 (1999).

23) Zhang R., Liu R., Liu Q. D., Mi H. Q., Wang N. S., Tradit. Chin. Drug Res. Pharmacol., 20, 338-341 (2009).

24) Montoro P., Carbone V., Quiroz Jde. D., De Simonel F., Pizza C., Phytochem. Anal., 15, 55-64 (2004). 